\title{
What Are the Changes and Burden Associated With COVID-19 in Diabetes Management and Delivery of the Diabetes Services?
}

\author{
Rohan Sadera $^{\text {a }}$, Joyce Youssef ${ }^{\mathrm{a}}$, Azza Ramadan ${ }^{\mathrm{b}}$, Mohamed H. Ahmed ${ }^{\mathrm{c}, \mathrm{d}}$
}

\begin{abstract}
Coronavirus disease 2019 (COVID-19) is an ongoing pandemic that has spread to almost all countries worldwide. Diabetes mellitus (DM) has been shown to increase the risk of COVID-19 disease severity. In this review, we provide a summary of the impact of COIVD-19 on diabetes management and related services. We searched the literature using the following databases: PubMed, Medline, Scopus and Google Scholar. These databases were searched using the keywords: COVID-19, diabetes type 1 and type 2. Lockdown causing social isolation and psychological issues, showed an influence on poor glycemic control amongst people with diabetes. Hence, the use of telemedicine and various technologies in the management of diabetes has substantially increased. In individuals with COVID-19, the current practice is not in favor of using sodium-glucose cotransporter 2 (SGLT2) inhibitors. Furthermore, insulin is recommended for the management of diabetes in critically ill patients. The COVID-19 pandemic was associated with many changes in diabetes management and the delivery of diabetes services.
\end{abstract}

Keywords: COVID-19; Diabetes services; Diabetes management; Telemedicine; Antidiabetic medication; Insulin

\section{Introduction}

Severe acute respiratory syndrome coronavirus 2 (SARSCoV-2), the viral illness known as coronavirus disease 2019 (COVID-19), was first identified in the city of Wuhan in China

Manuscript submitted September 23, 2020, accepted October 31, 2020

Published online December 22, 2020

${ }^{a}$ Medical School, University of Buckingham, Buckingham, UK ${ }^{b}$ College of Pharmacy, Al-Ain University, Abu Dhabi, United Arab Emirates 'Department of Medicine and HIV Metabolic Clinic, Milton Keynes University Hospital NHS Foundation Trust, Buckinghamshire, UK

${ }^{\mathrm{d} C}$ Corresponding Author: Mohamed H. Ahmed, Department of Medicine and HIV Metabolic Clinic, Milton Keynes University Hospital NHS Foundation Trust, Eaglestone, Milton Keynes, MK6 5LD, Buckinghamshire, UK. Email: Mohamed.Hassan-Ahmed@mkuh.nhs.uk

doi: https://doi.org/10.14740/jem699
$[1,2]$. It is a respiratory illness spread by airborne droplets from the respiratory tract [3]. Within this review, we aim to describe the impact of COVID-19 on diabetes mellitus (DM) management, treatment, diabetes services, and the current telemedicine practices.

\section{The Relationship Between DM and COVID-19}

DM alongside various other comorbidities is said to increase the risk of COVID-19 morbidity and mortality. The relationship between the two is complex, for instance, insulin resistance and hyperglycemia can increase synthesis of glycosylation end products (AGEs), oxidative stress, pro-inflammatory cytokines, and adhesion molecule production that stimulate tissue inflammation. This may lead to higher infections and worse outcomes especially with COVID-19 [4]. Poor glycemic control is another contribution to worse COVID-19 infection outcome. It has been linked to poor white cell function, including impaired lymphocyte proliferation in response to certain stimuli, and impaired monocytes/macrophages and neutrophils. In vitro research has shown increased influenza viral infection and replication, due to pulmonary cell exposure to high glucose concentrations that may apply to COVID-19 [4]. All of these can indicate a reason as to why morbidity and mortality is higher in diabetic individuals.

Angiotensin-converting enzyme 2 (ACE2) has been identified as a main receptor for COVID-19. It is widely expressed in the pancreas. It is hypothesised that COVID-19 can therefore damage the islets causing acute insulin-dependent DM. A study that compared 39 COVID-19 patients who never had diabetes to 39 matched healthy siblings showed that 20 out of the 39 COVID-19 patients developed diabetes during their

\section{Key Points}

- COVID-19 changed the use of certain antidiabetic medication.

- The benefits of telemedicine and online teaching need to be investigated.

- The psychological burden of COVID-19 needs further investigations.

- The burden on clinical chemistry laboratories also need to be investigated. 
hospitalisation. Hence, this pancreatic damage could also be present in diabetic patients infected with COVID-19, worsening their pancreatic function, which may explain their worsening morbidity and mortality. This needs to be researched further before conclusions are made [4].

Despite the link between COVID 19 and diabetes, the extent of this association is largely unknown. Because of this, in this article we aim to explore different aspects of COVID-19 and diabetes. From social isolation, technological advances, management changes to the virus' influence on diabetic emergencies. It will aim to give an overview of this important complicated interaction.

\section{Impact of Social Isolation on DM Patients}

During the COVID-19 pandemic, many countries went into lockdown, including the UK, to prevent the spread of COVID-19 $[5,6]$. However, the lockdown provided a lengthy period of social isolation [7]. In the Maastricht study [8], social isolation was shown to be associated with a greater prevalence of type 2 DM (T2DM). Lack of social participation was associated with pre-diabetes in both genders, and previously diagnosed diabetes in females. While living alone was also associated with previously diagnosed diabetes in males [8].

A narrative review carried out by Mohebi et al in 2013 [9], examined the effect of social isolation on diabetes management. It was concluded that social support increases selfcare diet, exercise, and adherence to management plans in people with T2DM. Also, increased social support decreased emotional distress from T2DM and improved the individuals' psychological well-being, which improved self-confidence, coping with the disease, and adherence to treatment [10]. On the other hand, lack of social support resulted in ignoring dietary recommendations and decreased self-care in individuals with diabetes [9]. Therefore, there is an association between good social support and positive outcomes in terms of disease management in individuals with diabetes.

Importantly, the COVID-19 pandemic has caused social isolation, especially for individuals with diabetes [11]. A narrative review conducted by Banerjee et al in 2020 [12] had shed light on the management of diabetes, specifically during the COVID-19 pandemic. The study illustrated the worsening of glycemic control in T2DM individuals during the outbreak. They associated poor glycemic control with social distancing and lockdown effects on lifestyles that restricted exercise, healthy food, difficulties in obtaining anti-diabetic medications, and lack of routine appointments regarding their diabetes.

Banerjee et al in 2020 [12] also examined several studies addressing the psychological health of DM patients during the pandemic. They reported that anxiety about the virus and the inability to meet loved ones had the most significant impact on mental health, which negatively affected DM self-management [12]. Furthermore, Hartmann-Boyce et al in 2020 [13] showed that social isolation during COVID-19 has a negative impact on mental health, which caused neglect of DM self-care and adherence to treatment. They also highlighted the issues regarding the lack of guidance in regard to patient's regular ap- pointments, foot checks, eye screening, and annual diabetes reviews. These have been postponed with no clear date as to when these services are to be running again [13]. Overall, the COVID-19 pandemic has caused a universal lack of social and healthcare support to individuals with diabetes.

\section{Role of Technology in Management of DM Dur- ing the COVID-19 Pandemic}

The role of technology in managing chronic diseases and diabetes at homes started to gain more attention during the pandemic of COVID-19 [14]. For instance, home urinalysis dipsticks were used to measure the urine albumin-to-creatinine ratio in Sussex Community Foundation NHS Trust. The results are then processed in a mobile app and sent to the diabetes team [15]. Free online courses (such as the ones offered by the University of Dundee) have been provided to educate patients about their T2DM. Other hospitals have continued to provide telephone consultations to diabetes patients, triaging the very high-risk to undergo screening and review. Diabetes Scotland has moved its on-site local groups to an online platform, allowing people with diabetes to discuss and share experiences, alongside offering a diabetes helpline [15]. In pediatric diabetology, pumps, smart pens and sensor data are all being used, and the results are then uploaded onto smartphones. These results are utilised for telemedicine consultations to aid in patient's DM management. Before the pandemic, diabetes management via online means was only thought to be a potential option given the savings in costs and time associated with it. However, due to the now greater need for these technologies, the pandemic has sped up the process to provide these services, where initial barriers such as data safety have become more relaxed [16]. And with the established success of continued glucose monitoring [17], which is a device placed under the skin to monitor blood glucose consistently, then displaying the results onto a device [18], it is no wonder that this is the direction of diabetic medicine. The previously mentioned technologies provide a future of easy and timely access of diabetes services for patients [19].

It is worth mentioning that there are still many obstacles that prevent the complete utilisation of these technologies. The main barriers are data security, data transfers and patient confidentiality [16]. Also, telemedicine currently involves a variety of different technologies and restrictive electronic health record policies. Hence, the utilisation of these various technologies and access to the restricted electronic records requires a high level of information technology (IT) skills from both the clinician's and patient's side [19]. Therefore, even though technology has played a significant role in diabetes management during the lockdown, many factors make face-to-face management a more favorable option.

\section{Telemedicine and Online Teaching's Role in DM Management Amid the COVID-19 Pandemic}

Telemedicine was in use before the COVID-19 pandemic. It 
utilises IT and communication systems currently in place to provide care and management to patients, especially for those in rural and remote settings. Therefore, it is not surprising that many health authorities, including the National Health Service (NHS) in the UK, used telemedicine during this pandemic, especially for outpatient follow-up [20, 21]. Other uses of telemedicine can be seen in (including pre COVID-19) electronic prescribing and retrieving patient records [22]. One of the UK's Department of Health aims is that by 2023 there will be an established electronic system where patients' records can be accessed all over UK hospitals [23, 24]. Different IT systems and financial readiness in different hospitals can represent a huge obstacle in connecting different hospitals [22]. Furthermore, telemedicine can be labour intensive [22]. However, given the increase in telemedicine usage during the COVID-19 pandemic, we may see significant changes in the direction of how diabetes services will be provided.

Even though digital resources have also been used for teaching purposes in healthcare environments and was found to be as effective as face-to-face teaching during the COVID-19 pandemic, more research is needed to evaluate its long-term effects on healthcare staff and patient outcomes especially for diabetes services [25]. The same evaluation is also needed for online learning in medical schools, as the long-term impact is not clear. This is especially important when teaching diabetes in medical school. It is worth mentioning that while all these measures are good substitutes, it does not replace the onsite essential patient experience for the health care professionals.

\section{The Use of Anti-Diabetic Medication for DM Management in COVID-19 Infected Individuals}

The wide use of ACE inhibitors (ACEIs) in individuals with diabetes who have hypertension was previously thought to increase the risk of COVID-19 infection by increasing the expression of ACE2. It was theorised that ACE2 is used as a receptor by the COVID-19 virus to facilitate its entry into pneumocytes [26, 27]. However, there has been no evidence to suggest that ACEIs or angiotensin receptor blockers (ARBs) increase the expression of ACE2 in the lung tissue [28]. Furthermore, the National Institute for Health and Care Excellence (NICE) and the World Health Organization suggest that patients on ACEIs or ARBs are not an increased risk of being infected with COVID-19 [29, 30]. Due to the lack of evidence, those who are on these medications are advised to continue taking them. As explained previously, ACE2 is also expressed within pancreatic cells, with certain groups of patients demonstrating a hyperglycemic state (without a diabetes diagnosis) [31]. Studies are being done to see the pathophysiology behind this phenomenon.

Statins are frequently used as part of diabetes management due to the general increased risk of cardiovascular disease and stroke [32]. It has been shown in the literature that they have the potential to reduce lung injury severity and mortality in those with COVID-19 infection. This is due to their immunomodulatory, anti-inflammatory, anti-thrombotic and antioxidant properties that may prevent lung injury due to excessive
ACE2. Therefore, those people with diabetes on statins should continue. But whether statins should be started during COVID-19 infection is something that needs more research before applied to clinical practice [33].

Metformin is the first line anti-diabetic medication used for managing T2DM. It has been previously shown that metformin doesn't affect ACE2 [34]. Hence, it may then still be used in diabetic patients who test positive for COVID-19. However, one of the side effects of metformin is lactic acidosis; therefore, it has been theorized to be discontinued in severe COVID-19 [35]. In severe COVID-19 infection, the hypoxic state may further exacerbate the risk of lactic acidosis. In contrast though, there has been research suggesting its antiinflammatory activity can reduce the risk of severe COVID-19 infection. This suggestion has been based on retrospective studies that have shown reduced mortality rates in metformin users in comparison to non-users [36]. For dipeptidyl peptidase 4 (DPP4) inhibitors, studies had indicated that it may reduce COVID-19 airway viral entry and replication. This is due to DPP4 being present throughout the respiratory tract, that may facilitate viral entry and the cytokine storm causing fatal COVID-19 pneumonia [37]. In regard to glucagon-like peptide 1 (GLP1) agonists, data are needed before their beneficial or harmful use in management of diabetes in patients who have the COVID-19 infection is determined [38-40].

Sulfonylureas have also been shown to have no effect on ACE2 [39]. Hence, they are currently recommended to be continued for use in diabetic patients. However, with the management of the COVID-19 pandemic, there have been issues in the disruption of the food supply. This may be particularly important in patients taking sulfonylureas as their inability to maintain nutrient intake may precipitate a hypoglycemic crisis. Therefore, the necessity of using Sulfonylureas in diabetic patients with COIVD-19 should be carefully evaluated or its use in diabetics must be carefully monitored.

Pioglitazone has been shown to increase the expression of ACE2 [34]. However, no evidence has been brought forward relating to pioglitazone's current use in diabetic patients with COVID-19. One study called for increased research into pioglitazone's use as supportive therapy for COVID-19 [41]. This was based on research results showing that pioglitazone caused a reduction in inflammatory markers [41] in addition to its use as anti-diabetes medication.

Regarding sodium-glucose cotransporter 2 (SGLT2) inhibitors, guidelines/recommendations have been put forth for their use in COVID-19 patients with type 1 and 2 diabetes. For clarification, SGLT2 inhibitors are not Food and Drug Administration (FDA)-approved for type 1 diabetes management in the USA [42], while in the UK the SGLT2 inhibitors can be used in type 1 as adjunct therapy to insulin [43]. It worth mentioning that, euglycemic diabetic ketoacidosis (DKA) can occur with use can occur with SGLT2 inhibitors use. COVID-19 infection appears to precipitate severe metabolic effects of diabetes, including DKA. Also, SGLT2 inhibitors can increase the risk of DKA. Currently, for patients with type 2 diabetes, clinicians may discontinue SGLT2 inhibitors due to the risk of masking the symptoms of DKA associated with COVID-19 [44]. Within T2DM, there is a risk that SGLT2 inhibitors can worsen DKA [35] and cause dehydration [39]. Hence, SGLT2 
inhibitors should be discontinued in patients with COVID-19 who are unable to maintain hydration and not eating (given that SGLT2 inhibitors promotes renal excretion of glucose).

Insulin is recommended for the management of diabetes in acute scenarios, such as in the critically ill diabetes patients. Due to the negative effects of discontinuing insulin, such as DKA, it is not recommended to stop insulin or exchange it for a different antidiabetic drug. However, increased monitoring must occur to ensure optimal blood glucose control and prevention of side effects of insulin therapy, such as hypoglycemia.

\section{Specific Management and Risk of Diabetes Emergencies}

It is vital to outline the management of diabetes in patient with COVID-19, as diabetes has shown to precipitate a more severe course of COVID-19, leading to the emergencies and complications [45]. The preventative measures of COVID-19 infection for diabetics are same as for the general public. These include frequently washing hands and adhering to the rules of social distancing [46]. Those with mild disease should be encouraged to stay at home and use paracetamol for symptomatic management [47]. A useful acronym provided by the Primary Care Diabetes Society for management of diabetics who fall ill due to another cause, is "SICK" (sugar, insulin, carbohydrates and ketones) [48]. The treatment should be according to the local hospital protocol for COVID-19 management [47, 49-51].

As stated before, insulin should be used for management of diabetes in critically ill patients. COVID-19 infection amongst individuals with diabetes can increase the risk of diabetes complications. For instance, one of the macrovascular complications of diabetes is the increased risk of ischemic heart disease, thrombotic events [52, 53], stroke and acute kidney injury (AKI) [54]. These risks combined with other higher incidence risks such as secondary hospital infections [55] means that utmost care must be taken when managing COVID-19 patients especially with comorbid diabetes. Monitoring and investigating for these complications may help to prevent further morbidities.

The presence of diabetes has shown to be a risk factor for severe illness and death within patients with COVID-19 [56]. There are three diabetic emergencies that may arise within diabetic patients with COVID-19. These are DKA, hyperosmolar hyperglycemic state (HHS) and hypoglycemia. These emergencies have become more prevalent within the COVID-19 pandemic due to factors such as social distancing, lack of access to health services and medication usage. Therefore, regular monitoring of glucose is highly recommended [4, 57-62].

Therefore, as mentioned earlier it is not surprising that the antidiabetic medication SGLT2 inhibitors were not recommended during COVID-19 as they may precipitate DKA. Metformin may lead to negative clinical outcomes in COVID-19 patients as it can precipitate lactic acidosis and kidney injury thus extra care is needed $[58,63]$. The presence of hyperglycemic complications in COVID-19 patients may also be used as a prognostic factor for the disease [64] and help to escalate

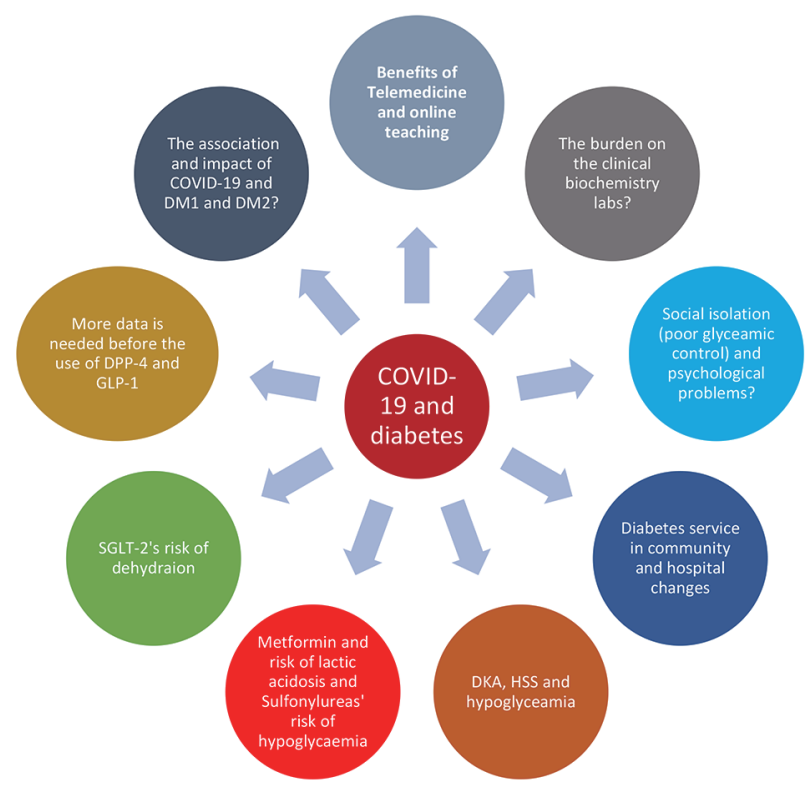

Figure 1. This diagrapm showed the multifaceted relationship between COVID-19 and diabetes management, pharmacotherapy, clinical chemistry laboratories and diabetes services. COVID-19: coronavirus disease 2019; DM: diabetes mellitus; DDP4: dipeptidyl peptidase 4; GLP1: glucagon-like peptide 1; SGLT2: sodium-glucose cotransporter 2; DKA: diabetic ketoacidosis; HHS: hyperosmolar hyperglycemic state.

management with limited evidence suggesting that a prompt correction of hyperglycemia may help management [64].

The hypoglycemia that occurs in diabetic patients may be secondary to infections [65] but can also be due to diabetes medication and insulin. Care must also be taken when administering chloroquine to diabetic patients due to the risk of precipitating hypoglycemia [66] which has shown to increase death rates in patients with pneumonia [67], (a potential complication of COVID-19). Hypoglycemia may also warrant the need to look for adrenal insufficiency (especially in vasopressorresistant hypotension) which can also exist with COVID-19 [68]. Limited case reports have shown that COVID-19 may cause adrenal insufficiency, but this is a rare complication of the infection [69]. Lastly, one must remember that T1DM may coexist with adrenal insufficiency in a patient.

\section{Conclusions}

COVID-19 has changed diabetes management and the use of anti-diabetic medication. Also, significant changes have occurred in the way that the diabetes service is delivered. Overall, we have addressed several topics that explored the relationships between COVID-19 and DM management, treatment and related technologies (Fig. 1). Several challenges and questions about COVID-19 and diabetes remain outstanding and merits further investigation (Fig. 1). Among these questions are: 1) Why is DM a risk factor for severe COVID-19 morbidity and mortality? 2) What is the direct and indirect impact of COVID-19 on the pancreas? 3) Will patients with type 1 dia- 
betes, an autoimmune disease, spared from infection of COVID-19? [70]. Also, the psychological burden of COVID-19 in individuals with diabetes need to be further researched. Lastly, further research is needed to assess the advantages and drawbacks of the current management measures in optimising diabetes control and decreasing diabetes complications.

\section{Acknowledgments}

The authors acknowledge the support of their families during the time of writing this review.

\section{Financial Disclosure}

None to declare.

\section{Conflict of Interest}

Rohan Sadera, Joyce Youssef, Azza Ramadan and Mohamed $\mathrm{H}$. Ahmed declare that they have no conflict of interest.

\section{Informed Consent}

Not applicable.

\section{Author Contributions}

Mohamed H. Ahmed conceptualized and designed the study. Mohamed H. Ahmed and Azza Ramadan contributed to the administrative support. All the authors involved in provision of study materials, collection and assembly of data, data analysis and interpretation, and manuscript writing. All the authors did the final approval of manuscript.

\section{Data Availability}

The authors declare that data supporting the findings of this study are available within the article.

\section{References}

1. Mazucanti CH, Egan JM. SARS-CoV-2 disease severity and diabetes: why the connection and what is to be done? Immun Ageing. 2020;17:21.

2. Andersen KG, Rambaut A, Lipkin WI, Holmes EC, Garry RF. The proximal origin of SARS-CoV-2. Nat Med. 2020;26(4):450-452.

3. WHO. 2020. Transmission Of SARS-Cov-2: Implications for infection prevention precautions. [online] Available at: https://www.who.int/news-room/commentaries/detail/ transmission-of-sars-cov-2-implications-for-infection- prevention-precautions. [Accessed October 26, 2020].

4. Hussain A, Bhowmik B, do Vale Moreira NC. COVID-19 and diabetes: knowledge in progress. Diabetes Res Clin Pract. 2020;162:108142.

5. BBC News. Do British people still accept the lockdown? 2020. [online] Available at: https://www.bbc.co.uk/news/ uk-52495201. [Accessed May 22, 2020].

6. Carroll L. UK coronavirus lockdown: what you can and cannot do. [online] The Guardian. 2020. Available at: https://www.theguardian.com/world/2020/mar/25/ukcoronavirus-lockdown-rules-what-you-can-and-cannotdo. [Accessed May 22, 2020].

7. Walker P. Coronavirus lockdown: what are the new rules announced by Boris Johnson? 2020. [online] the Guardian. Available at: https://www.theguardian.com/ world/2020/may/10/uk-coronavirus-lockdown-what-hasboris-johnson-announced [Accessed May 22, 2020].

8. Brinkhues S, Dukers-Muijrers N, Hoebe C, van der Kallen CJH, Dagnelie PC, Koster A, Henry RMA, et al. Socially isolated individuals are more prone to have newly diagnosed and prevalent type 2 diabetes mellitus - the Maastricht study. BMC Public Health. 2017;17(1):955.

9. Rad GS, Bakht LA, Feizi A, Mohebi S. Importance of social support in diabetes care. J Educ Health Promot. 2013;2:62.

10. Ramkisson S, Pillay BJ, Sibanda W. Social support and coping in adults with type 2 diabetes. Afr J Prim Health Care Fam Med. 2017;9(1):e1-e8.

11. Wilder-Smith A, Freedman DO. Isolation, quarantine, social distancing and community containment: pivotal role for old-style public health measures in the novel coronavirus (2019-nCoV) outbreak. J Travel Med. 2020;27(2):taaa020.

12. Banerjee M, Chakraborty S, Pal R. Diabetes self-management amid COVID-19 pandemic. Diabetes Metab Syndr. 2020;14(4):351-354.

13. Hartmann-Boyce J, Morris E, Goyder C, Kinton J, Perring J, Nunan D, Khunti K. Managing diabetes during the COVID-19 pandemic - CEBM. [online] CEBM. 2020. Available at: https://www.cebm.net/covid-19/managingdiabetes-during-the-covid-19-pandemic/. Accessed May 22, 2020.

14. NHS England. 2020. [online] Available at: https:// www.england.nhs.uk/coronavirus/wp-content/uploads/ sites/52/2020/03/speciality-guide-diabetes-19-march-v2updated.pdf [Accessed May 25, 2020].

15. Ranscombe P. How diabetes management is adapting amid the COVID-19 pandemic. Lancet Diabetes Endocrinol. 2020;8(7):571.

16. Danne T, Limbert C. COVID-19, type 1 diabetes, and technology: why paediatric patients are leading the way. Lancet Diabetes Endocrinol. 2020;8(6):465-467.

17. Rodbard D. Continuous glucose monitoring: a review of successes, challenges, and opportunities. Diabetes Technol Ther. 2016;18(Suppl 2):S3-S13.

18. National Institute of Diabetes and Digestive and Kidney Diseases. 2017. Continuous Glucose Monitoring | NIDDK. [online] Available at: https://www.niddk.nih. gov/health-information/diabetes/overview/managing- 
diabetes/continuous-glucose-monitoring\#: : text=A\%20 CGM\%20works\%20through\%20a,the\%20fluid $\% 20$ between $\% 20$ the $\% 20$ cells. [Accessed October 27, 2020].

19. Gamble A, Pham Q, Goyal S, Cafazzo JA. The challenges of COVID-19 for people living with diabetes: considerations for digital health. JMIR Diabetes. 2020;5(2):e19581.

20. Zhang XY, Zhang P. Telemedicine in clinical setting. Exp Ther Med. 2016;12(4):2405-2407.

21. Debnath D. Activity analysis of telemedicine in the UK. Postgrad Med J. 2004;80(944):335-338.

22. Asthana S, Jones R, Sheaff R. Why does the NHS struggle to adopt eHealth innovations? A review of macro, meso and micro factors. BMC Health Serv Res. 2019;19(1):984.

23. HM government, 2014. Personalised health and care. 2020. [ebook] UK. Available at: https://assets.publishing.service.gov.uk/government/uploads/system/uploads/ attachment_data/file/384650/NIB_Report.pdf [Accessed May 27, 2020].

24. Johnston DS. Digital maturity: are we ready to use technology in the NHS? Future Healthc J. 2017;4(3):189-192.

25. Lawn S, Zhi X, Morello A. An integrative review of e-learning in the delivery of self-management support training for health professionals. BMC Med Educ. 2017;17(1):183.

26. Pal R, Bhansali A. COVID-19, diabetes mellitus and ACE2: The conundrum. Diabetes Res Clin Pract. 2020;162:108132.

27. Fang L, Karakiulakis G, Roth M. Are patients with hypertension and diabetes mellitus at increased risk for COVID-19 infection? Lancet Respir Med. 2020;8(4):e21.

28. South AM, Brady TM, Flynn JT. ACE2 (AngiotensinConverting Enzyme 2), COVID-19, and ACE Inhibitor and Ang II (Angiotensin II) receptor blocker use during the pandemic: the pediatric perspective. Hypertension. 2020;76(1):16-22.

29. Anon, 2020. Key Messages | COVID-19 rapid evidence summary: angiotensin-converting enzyme inhibitors (Aceis) or angiotensin receptor blockers (Arbs) in people with or at risk of COVID-19 | Advice | NICE. [online] Nice.org.uk. Available at: https://www.nice.org.uk/ advice/es24/chapter/Key-messages [Accessed June 1, 2020].

30. Anon, 2020. COVID-19 and the use of angiotensin-converting enzyme inhibitors and receptor blockers. [online] Who.int. Available at: https://www.who.int/news-room/ commentaries/detail/covid-19-and-the-use-of-angiotensin-converting-enzyme-inhibitors-and-receptor-blockers [Accessed June 1, 2020].

31. Thaweerat W. Current evidence on pancreatic involvement in SARS-CoV-2 infection. Pancreatology. 2020;20(5):1013-1014.

32. Matthews DA, Voytovich AE. An appraisal of goals for residency training in internal medicine. J Med Educ. 1985;60(7):536-544.

33. Diabetes. 2019. Diabetes and statins have a complex relationship and are the focus of intense patient and healthcare debate. Statins Are Cholesterol-Lowering Drugs. [online] Available at: https://www.diabetes.co.uk/diabetes-medication/diabetes-and-statins.html [Accessed Oc- tober 27, 2020].

34. Pal R, Bhadada SK. Reply to comment on "Should antidiabetic medications be reconsidered amid COVID-19 pandemic?". Diabetes Res Clin Pract. 2020;164:108192.

35. Orioli L, Hermans MP, Thissen JP, Maiter D, Vandeleene B, Yombi JC. COVID-19 in diabetic patients: Related risks and specifics of management. Ann Endocrinol (Paris). $2020 ; 81(2-3): 101-109$.

36. Scheen AJ. Metformin and COVID-19: From cellular mechanisms to reduced mortality. Diabetes Metab. 2020.

37. Solerte SB, Di Sabatino A, Galli M, Fiorina P. Dipeptidyl peptidase-4 (DPP4) inhibition in COVID-19. Acta Diabetol. 2020;57(7):779-783.

38. Anon, 2020. Expert panel offers practical recommendations for diabetes management during coronavirus pandemic. [online] Mims.co.uk. Available at: https://www. mims.co.uk/expert-panel-offers-practical-recommendations-diabetes-management-during-coronavirus-pandemic/diabetes/article/1681754 [Accessed May 25, 2020].

39. Katulanda P, Dissanayake HA, Ranathunga I, Ratnasamy V, Wijewickrama PSA, Yogendranathan N, Gamage KKK, et al. Prevention and management of COVID-19 among patients with diabetes: an appraisal of the literature. Diabetologia. 2020;63(8):1440-1452.

40. Dalan R. Is DPP4 inhibition a comrade or adversary in COVID-19 infection. Diabetes Res Clin Pract. 2020;164:108216.

41. Carboni E, Carta AR, Carboni E. Can pioglitazone be potentially useful therapeutically in treating patients with COVID-19? Med Hypotheses. 2020;140:109776.

42. Diabetes. 2019. FDA rejects approval of empagliflozin for type 1 diabetes - Diabetes. [online] Available at: https://www.diabetes.co.uk/news/2019/Nov/fdarejects-approval-of-empagliflozin-for-type-1-diabetes.html\#: : text $=$ However $\% 2 \mathrm{C} \% 20$ the $\% 20$ US $\% 20$ FDA\%20has,DKA\%20in\%20type\%201\%20diabetes. [Accessed October 27, 2020].

43. NICE. 2020. Dapagliflozin | Drug | BNF Content Published By NICE. [online] Bnf.nice.org.uk. Available at: https://bnf.nice.org.uk/drug/dapagliflozin.html [Accessed October 27, 2020].

44. Anon. 2020. Updates: coronavirus and diabetes. [online] Diabetes UK. Available at: https://www.diabetes.org.uk/ about_us/news/coronavirus [Accessed May 25, 2020].

45. Targher G, Mantovani A, Wang XB, Yan HD, Sun QF, Pan KH, Byrne CD, et al. Patients with diabetes are at higher risk for severe illness from COVID-19. Diabetes Metab. 2020;46(4):335-337.

46. Anon. 2020. COVID-19 and diabetes. [online] Idf.org. Available at: https://www.idf.org/aboutdiabetes/what-isdiabetes/covid-19-and-diabetes/1-covid-19-and-diabetes. html [Accessed May 25, 2020].

47. Gupta R, Ghosh A, Singh AK, Misra A. Clinical considerations for patients with diabetes in times of COVID-19 epidemic. Diabetes Metab Syndr. 2020;14(3):211-212.

48. Anon. 2020. Coronavirus: specific advice for people living with diabetes | Know Diabetes. [online] knowdiabetes.org.uk. Available at: https://www.knowdiabetes.org. uk/for-you/covid-19-staying-healthy/coronavirus-spe- 
cific-advice-for-people-living-with-diabetes/ [Accessed May 25, 2020].

49. Chen CY, Wang FL, Lin CC. Chronic hydroxychloroquine use associated with QT prolongation and refractory ventricular arrhythmia. Clin Toxicol (Phila). 2006;44(2):173175.

50. Mehra M, Desai S, Ruschitzka F, Patel A. Hydroxychloroquine or chloroquine with or without a macrolide for treatment of COVID-19: a multinational registry analysis. The Lancet. 2020.

51. Anesi G. Coronavirus disease 2019 (COVID-19): critical care and airway management issues. [online] Uptodate.com. 2020. Available at: https://www.uptodate.com/ contents/coronavirus-disease-2019-covid-19-criticalcare-and-airway-management-issues [Accessed May 25, 2020].

52. Klok FA, Kruip M, van der Meer NJM, Arbous MS, Gommers D, Kant KM, Kaptein FHJ, et al. Confirmation of the high cumulative incidence of thrombotic complications in critically ill ICU patients with COVID-19: An updated analysis. Thromb Res. 2020;191:148-150.

53. Helms J, Tacquard C, Severac F, Leonard-Lorant I, Ohana $\mathrm{M}$, Delabranche X, Merdji H, et al. High risk of thrombosis in patients with severe SARS-CoV-2 infection: a multicenter prospective cohort study. Intensive Care Med. 2020;46(6):1089-1098.

54. Naicker S, Yang CW, Hwang SJ, Liu BC, Chen JH, Jha V. The Novel Coronavirus 2019 epidemic and kidneys. Kidney Int. 2020;97(5):824-828.

55. Rodriguez-Morales AJ, Cardona-Ospina JA, Gutierrez-Ocampo E, Villamizar-Pena R, Holguin-Rivera Y, Escalera-Antezana JP, Alvarado-Arnez LE, et al. Clinical, laboratory and imaging features of COVID-19: A systematic review and meta-analysis. Travel Med Infect Dis. 2020;34:101623.

56. Muniyappa R, Gubbi S. COVID-19 pandemic, coronaviruses, and diabetes mellitus. Am J Physiol Endocrinol Metab. 2020;318(5):E736-E741.

57. Simonnet A, Chetboun M, Poissy J, Raverdy V, Noulette J, Duhamel A, Labreuche J, et al. High prevalence of obesity in severe acute respiratory syndrome coronavirus-2 (SARS-CoV-2) requiring invasive mechanical ventilation. Obesity (Silver Spring). 2020;28(7):1195-1199.

58. Diabetes.org.uk. 2020. Concise advice on inpatient diabetes (COVID: Diabetes): front door guidance. [online] Available at: https://www.diabetes.org.uk/resources-s3/ public/2020-04/COvID Front Door v1.0.pdf [Accessed
May 16, 2020].

59. Birmingham Health Partners. COVID-19 Research Briefing 29/04/20 - Diabetes - Birmingham Health Partners. 2020. [online] Birminghamhealthpartners.co.uk. Available at: https://www.birminghamhealthpartners.co.uk/ covid-19-research-briefing-29-04-20-diabetes/ [Accessed May 16, 2020].

60. Tidy C. Diabetic ketoacidosis. Information about DKA. Patient. [online] Patient.info. 2016. Available at: https:// patient.info/doctor/diabetic-ketoacidosis [Accessed May 16, 2020].

61. Li J, Wang X, Chen J, Zuo X, Zhang H, Deng A. COVID-19 infection may cause ketosis and ketoacidosis. Diabetes Obes Metab. 2020.

62. Yang JK, Lin SS, Ji XJ, Guo LM. Binding of SARS coronavirus to its receptor damages islets and causes acute diabetes. Acta Diabetol. 2010;47(3):193-199.

63. Bornstein SR, Rubino F, Khunti K, Mingrone G, Hopkins D, Birkenfeld AL, Boehm B, et al. Practical recommendations for the management of diabetes in patients with COVID-19. Lancet Diabetes Endocrinol. 2020;8(6):546550.

64. Ceriello A. Hyperglycemia and the worse prognosis of COVID-19. Why a fast blood glucose control should be mandatory. Diabetes Res Clin Pract. 2020;163:108186.

65. Casqueiro J, Casqueiro J, Alves C. Infections in patients with diabetes mellitus: A review of pathogenesis. Indian J Endocrinol Metab. 2012;16(Suppl 1):S27-36.

66. Anon ND. CHLOROQUINE | Drug | BNF Content Published By NICE. [online] Bnf.nice.org.uk. Available at: https://bnf.nice.org.uk/drug/chloroquine.html [Accessed May 16, 2020].

67. Mortensen EM, Garcia S, Leykum L, Nakashima B, Restrepo MI, Anzueto A. Association of hypoglycemia with mortality for subjects hospitalized with pneumonia. Am J Med Sci. 2010;339(3):239-243.

68. Scaroni C, Armigliato M, Cannavo S. COVID-19 outbreak and steroids administration: are patients treated for Sars-Cov-2 at risk of adrenal insufficiency? J Endocrinol Invest. 2020;43(7):1035-1036.

69. Heidarpour M, Vakhshoori M, Abbasi S, Shafie D, Rezaei N. Adrenal insufficiency in coronavirus disease 2019: a case report. J Med Case Rep. 2020;14(1):134.

70. Tatti P, Tonolo G, Zanfardino A, Iafusco D. Is it fair to hope that patients with Type 1 Diabetes (autoimmune) may be spared by the infection of Covid-19? Med Hypotheses. 2020;142:109795. 